\title{
MESHLESS COLLOCATION METHODS FOR SOLVING PDES ON SURFACES
}

\author{
MENG CHEN ${ }^{1}$, KA CHUN CHEUNG $^{2}$ \& LEEVAN LING ${ }^{3}$ \\ ${ }^{1}$ ASTRI Hong Kong Applied Science and Technology Research Institute Company Limited, Hong Kong \\ ${ }^{2}$ NVIDIA AI Technology Center (NVAITC), NVIDIA, USA \\ ${ }^{3}$ Department of Mathematics, Hong Kong Baptist University, Hong Kong
}

\begin{abstract}
We present three recently proposed kernel-based collocation methods in unified notations as an easy reference for practitioners who need to solve PDEs on surfaces $\mathcal{S} \subset \mathbb{R}^{d}$. These PDEs closely resemble their Euclidean counterparts, except that the problem domains change from bulk regions with a flat geometry of some surfaces, on which curvatures play an important role in the physical processes. First, we present a formulation to solve surface PDEs in a narrow band domain containing the surface. This class of numerical methods is known as the embedding types. Next, we present another formulation that works solely on the surface, which is commonly referred to as the intrinsic approach. Convergent estimates and numerical examples for both formulations will be given. For the latter, we solve both the linear and nonlinear time-dependent parabolic equations on static and moving surfaces.

Keywords: kernel-based collocation methods, elliptic partial differential equations on manifolds, convergence estimate.
\end{abstract}

\section{INTRODUCTION}

Throughout the paper, we use $\mathcal{S} \subset \mathbb{R}^{d}$ to denote some sufficiently smooth, connected, and compact surface with bounded geometry with $\operatorname{dim}(\mathcal{S})=d-1$. All surface functions and operators are labelled with subscripts $\mathcal{S}$ for clarity. In particular, the surface gradient $\nabla_{\mathcal{S}}$ and the Laplace-Beltrami $\Delta_{\mathcal{S}}$ operators (a.k.a. the surface Laplacian) can be defined in terms of the standard Euclidean gradient $\nabla$ and Laplacian $\Delta$ operators for $\mathbb{R}^{d}$ via projections:

$$
\nabla_{\mathcal{S}}:=\left(I-\mathbf{n n}^{T}\right) \nabla \quad \text { and } \quad \Delta_{\mathcal{S}}:=\nabla_{\mathcal{S}} \cdot \nabla_{\mathcal{S}}
$$

where $I$ is the identity operator and $\mathbf{n}=\mathbf{n}(\boldsymbol{\xi})$ denote the unit outward normal vector at $\boldsymbol{\xi} \in \mathcal{S}$. For bounded Euclidian domains $\Omega$, we work on the standard Hilbert spaces $H^{m}(\Omega)$ and $H^{m-1 / 2}(\Omega)$ with standard Sobolev norms for some integer $m \geq d / 2$. Sobolev spaces on surfaces can be defined by $H^{m}(\mathcal{S}):=\left(I-\Delta_{\mathcal{S}}\right)^{-m / 2} L^{2}(\mathcal{S})$, see [1].

The types of partial differential equations (PDEs) that we concerned are time-independent strongly elliptic equations on surfaces $\mathcal{S} \subset \mathbb{R}^{d}$ in the form of

$$
\mathcal{L}_{\mathcal{S}} u_{\mathcal{S}}:=\left(-a_{\mathcal{S}} \Delta_{\mathcal{S}}+\mathbf{b}_{\mathcal{S}} \cdot \nabla_{\mathcal{S}}+c_{\mathcal{S}}\right) u_{\mathcal{S}}=f_{\mathcal{S}}
$$

for some surface differential operator $\mathcal{L}_{\mathcal{S}}: H^{m}(\mathcal{S}) \rightarrow H^{m-2}(\mathcal{S})$ with $W_{\infty}^{m}(\mathcal{S})$-bounded coefficients $a_{\mathcal{S}}, c_{\mathcal{S}}: \mathcal{S} \rightarrow \mathbb{R}$, and $\mathbf{b}_{\mathcal{S}}: \mathcal{S} \rightarrow \mathbb{R}^{d}$. We assume the existence [2] of classical solutions $u_{\mathcal{S}}^{*}$ to (2) in Hilbert spaces $H^{m}(\mathcal{S})$.

\section{EMBEDDING KANSA METHODS}

Methods in this section are variants of Kansa methods [3], [4] that built upon some constantalong-normal property. They are generalization of the finite difference based closest point method [5] and its meshfree extension [6]. Firstly, we define the closest point mapping cp

$$
\operatorname{cp}(\mathbf{x})=\underset{\boldsymbol{\xi} \in \mathcal{S}}{\arg \inf }\|\boldsymbol{\xi}-\mathbf{x}\|_{\ell^{2}\left(\mathbb{R}^{d}\right)},
$$


which maps each near-surface point $\mathbf{x} \in \mathbb{R}^{d}$ onto the nearest surface $\operatorname{cp}(\mathbf{x}) \in \mathcal{S}$. For any smooth surface $\mathcal{S}$ of class $C^{m+1}$, we know that [7] the cp map is well-defined in some narrow-band domain containing $\mathcal{S}$

$$
\Omega=\Omega_{\delta}:=\left\{\mathbf{x} \in \mathbb{R}^{d}: \inf _{\boldsymbol{\xi} \in \mathcal{S}}\|\boldsymbol{\xi}-\mathbf{x}\|_{\ell^{2}\left(\mathbb{R}^{d}\right)}<\delta\right\}
$$

for some constant $\delta$ depending only on $\mathcal{S}$ and $m$ such that $u_{\mathcal{S}} \circ \mathrm{cp} \in H^{m}(\Omega)$ for all $u_{\mathcal{S}} \in H^{m}(\mathcal{S})$.

An embedded PDE associated with (2) is in the form of

$$
\mathcal{L}:=-a \Delta+\mathbf{b} \cdot \nabla+c \text { in } \Omega,
$$

with all coefficients in (4) being the cp-extensions of those in (2), i.e. $a=a_{\mathcal{S}} \circ \mathrm{cp}$ and etc. for all $\mathrm{x} \in \Omega$. The following theorem gives the relationship of operators on $\mathcal{S}$ and $\Omega$, and the solutions of (2) and (4).

Theorem 1. [8, Thm. 2.1] Let $\mathcal{S} \subset \mathbb{R}^{d}$ be a codimension one $C^{3}$-smooth, connected and compact surface with well-defined normal $\mathbf{n}=\mathbf{n}(\mathbf{x})$ for all $\mathbf{x} \in \mathcal{S}$. Let $u \in C^{2}(\Omega) \cap$ $H^{2+1 / 2}(\Omega)$ be an extension of $u_{\mathcal{S}} \in H^{2}(\mathcal{S})$ with $u_{\mid \mathcal{S}}=u_{\mathcal{S}}$. Then,

$$
\nabla_{\mathcal{S}} u:=\nabla u-\mathbf{n} \partial_{\mathbf{n}} u \quad \text { and } \quad \Delta_{\mathcal{S}} u:=\Delta u-H_{\mathcal{S}} \partial_{\mathbf{n}} u-\partial_{\mathbf{n}}^{(2)} u \quad \text { on } \mathcal{S},
$$

where $\partial_{\mathbf{n}} u:=\mathbf{n}^{T} \nabla u, \partial_{\mathbf{n}}^{(2)} u:=\mathbf{n}^{T} J(\nabla u) \mathbf{n}$ and $H_{\mathcal{S}}(\boldsymbol{\xi})=\operatorname{trace}\left(J(\mathbf{n})\left(I-\mathbf{n n}^{T}\right)\right)$, which is $d$ times the mean curvature of $\mathcal{S}$ at $\mathbf{x}$, defined using the Jacobian operator $J$ in Euclidean space. In particular, for any second order differential operator in the form of (2), if u satisfies the embedding conditions

$$
\partial_{\mathbf{n}} u=0 \quad \text { and } \quad \partial_{\mathbf{n}}^{(2)} u=0 \quad \text { on } \mathcal{S},
$$

then $\mathcal{L}_{\mathcal{S}} u_{\mathcal{S}}=\mathcal{L}$ u on $\mathcal{S}$.

Note that (4) is simply an elliptic PDE posed in the narrow-band domain $\Omega$. Once we solve (4) for $u$ with constant-along-normal property, restricting it to the surface yields the surface solution $u_{\mathcal{S}}=u_{\mid \mathcal{S}}$.

\subsection{Numerical algorithms}

For the sake of convergence proof, we use kernels $\Phi_{m}: \mathbb{R}^{d} \times \mathbb{R}^{d} \rightarrow \mathbb{R}$ that are translationinvariant, symmetric positive definite with smoothness $m$, and satisfy

$$
c\left(1+\|\omega\|_{2}^{2}\right)^{-m} \leq \widehat{\Phi_{m}}(\omega) \leq C\left(1+\|\omega\|_{2}^{2}\right)^{-m} \quad \text { for all } \omega \in \mathbb{R}^{d},
$$

for some constants $0<c \leq C$. Commonly used kernels of this sort includes the standard Whittle-Matérn-Sobolev kernels, that are defined via the Bessel functions of the second kind in the form of

$$
\Phi_{m}(\mathbf{x}):=\|\mathbf{x}\|_{2}^{m-\frac{d}{2}} \mathcal{K}_{m-\frac{d}{2}}\left(\|\mathbf{x}\|_{2}\right) \quad \text { for all } x \in \mathbb{R}^{d},
$$

and the class of compactly supported piecewise polynomial Wendland functions [9].

Let $Z=\left\{z_{1}, \ldots, z_{n_{Z}}\right\} \subset \Omega_{\delta}$ denote a discrete set of trial centers in the narrow-band domain with radius $\delta$ in (3) and we define the corresponding finite-dimensional trial space by

$$
\mathcal{U}_{Z, \Omega, \Phi_{m}}:=\operatorname{span}\left\{\Phi_{m}\left(\cdot-z_{j}\right): z_{j} \in Z\right\}
$$


that is a subspace of the native space $\mathcal{N}_{\Omega, \Phi_{m}}$ of $\Phi_{m}$ [10], [11]. On the test side, we pick any discrete set $X=\left\{x_{1}, \ldots, x_{n_{X}}\right\} \subset \mathcal{S}$ of sufficiently dense collocation points (with respect to $Z$ ) on the surface.

Under this surface-version setup for overdetermined Kansa methods [12], a convergent numerical solution [8, Thm. 4.1] is defined by

$$
\underset{u \in \mathcal{U}_{Z, \Omega_{\delta}, \Phi_{m}}}{\arg \inf }(\underbrace{\|\mathcal{L} u-f\|_{\ell^{2}(X)}^{2}}_{\text {PDE collocations }}+\underbrace{\left\|\partial_{\mathbf{n}} u\right\|_{\ell^{2}(X)}^{2}+\left\|\partial_{\mathbf{n}}^{(2)} u\right\|_{\ell^{2}(X)}^{2}}_{\text {Embedding conditions }}+h_{X}^{\epsilon} \underbrace{\left\|u-u_{\mid \mathcal{S}} \circ \mathrm{cp}\right\|_{\ell^{2}(Z)}^{2}}_{\text {Stability conditions }}),
$$

for some $m>5 / 2+d / 2, \epsilon>0$, and with the mesh norm of $X$ defined by the shortest distance function $d_{\mathcal{S}}: \mathcal{S} \times \mathcal{S} \rightarrow \mathbb{R}$ on $\mathcal{S}$ as

$$
h_{X}:=\sup _{\xi \in \mathcal{S}} \min _{x \in X} d_{\mathcal{S}}(x, \xi) .
$$

Note that the Euclidean differential operator $\mathcal{L}$ in (9) is evaluated only at $X \subset \mathcal{S}$. It is unnecessary to cp-extend any coefficients of $\mathcal{L}_{\mathcal{S}}$ away from $\mathcal{S}$. The $\left(3 n_{X}+n_{Z}\right) \times n_{Z}$ overdetermined linear system in (9) can be expressed in matrix form as

$$
\left(\begin{array}{c}
\mathcal{L} \Phi_{m}(X, Z) \\
\partial_{\mathbf{n}} \Phi_{m}(X, Z) \\
\partial_{\mathbf{n}}^{(2)} \Phi_{m}(X, Z) \\
h_{X}^{\epsilon}\left(\Phi_{m}(Z \backslash \mathcal{S}, Z)-\Phi_{m}(\operatorname{cp}(Z \backslash \mathcal{S}), Z)\right)
\end{array}\right) \boldsymbol{\lambda}=\left(\begin{array}{c}
f(X) \\
\mathbf{0} \\
\mathbf{0} \\
\mathbf{0}
\end{array}\right)
$$

in which all differential operators act on the first argument of $\Phi_{m}$. Solving (10) in the leastsquares sense yields the unknown coefficient $\boldsymbol{\lambda}=\left\{\lambda_{i}\right\}_{i=1}^{n_{Z}}$. The Kansa solution can then be evaluated everywhere on $\mathcal{S}$ by

$$
U=\sum_{\zeta_{i} \in Z} \lambda_{i} \Phi_{m}\left(\cdot, \zeta_{i}\right)
$$

An error estimate for this solution is

$$
\begin{aligned}
& \left\|U-u_{\mathcal{S}}^{*}\right\|_{H^{k}(\mathcal{S})} \\
& \quad \leq C\left(h_{Z}^{m-k-1 / 2}+\delta^{1 / 2} h_{X}^{2-k} h_{Z}^{m-2-d / 2}+\delta h_{X}^{d / 2-k+3 / 2+\epsilon} h_{Z}^{m-d}\right)\left\|u_{\mathcal{S}}^{*}\right\|_{H^{m}(\mathcal{S})}
\end{aligned}
$$

for any $k \geq 2$ and $m>k+d / 2+3 / 2$. By examining the right-hand side of this estimate, we see that using $\delta \approx h_{Z}$ is a reasonable choice for the radius of the narrow-band domain $\Omega$.

Numerical evidence in [8] suggests that a reduced system by dropping the stability conditions in (9) has better numerically robustness when the set of collocation points $X$ is not dense enough to fulfil the theoretical requirement, i.e., when $h_{X}$ is larger then the theory allows. That is, we can solve the reduced $3 n_{X} \times n_{Z}$ overdetermined linear system

$$
\left(\begin{array}{c}
\mathcal{L} \Phi_{m}(X, Z) \\
\partial_{\mathbf{n}} \Phi_{m}(X, Z) \\
\partial_{\mathbf{n}}^{(2)} \Phi_{m}(X, Z)
\end{array}\right) \boldsymbol{\lambda}=\left(\begin{array}{c}
f(X) \\
\mathbf{0} \\
\mathbf{0}
\end{array}\right)
$$

for $\boldsymbol{\lambda}$ instead. In cases when the convergent theory applies, accuracy of (10) and (13) are nearly identical. For this reason, our numerical demonstration in the latter section will focus on the reduced system (13) instead. 


\section{INTRINSIC KANSA METHODS}

Let the notation $Z$ be redefined and reused. In this section, we are going to use a trial space defined solely on the surface $\mathcal{S}$ (instead of in some narrow-banded domain). Thus, we need a set of trial centers $Z=\left\{z_{1}, \ldots, z_{n_{Z}}\right\} \subset \mathcal{S}$ to be defined on the surface. We still require the kernel to satisfy the decay condition (7) with smoothness order $m$.

Under the assumption in [13], [14] that $m>d / 2$, we can obtain surface kernels

$$
\Psi_{m-1 / 2}(\cdot, \cdot):=\Phi_{m}(\cdot, \cdot)_{\mid \mathcal{S} \times \mathcal{S}}: \mathcal{S} \times \mathcal{S} \rightarrow \mathbb{R}
$$

that reproduce $H^{m-1 / 2}(\mathcal{S})$ simply by restricting the global kernels $\Phi_{m}$ on $\mathcal{S}$. The surface kernel here severs theoretical purpose and, in actual implementation, we are using the global kernel $\Phi_{m}$ of our choice.

Within the corresponding trial space, which is the span of translations of $\Psi_{m-1 / 2}$, i.e.,

$$
\mathcal{U}_{Z, \mathcal{S}, \Psi_{m-1 / 2}}:=\operatorname{span}\left\{\Phi_{m}(\cdot-\boldsymbol{\xi}) \mid \boldsymbol{\xi} \in Z\right\},
$$

we define an intrinsic least-squares solution by

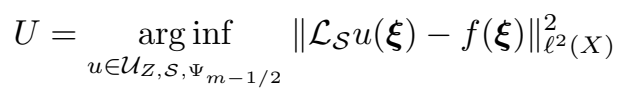

for some sufficiently dense set of collocation points $X \subset \mathcal{S}$. In [15], we show that the numerical solution $U$ of (15) satisfies the estimate

$$
\left\|U-u_{\mathcal{S}}^{*}\right\|_{H^{k}(\mathcal{S})} \leq C h_{Z}^{m-d / 2}\left(h_{Z}^{-k}+h_{X}^{-k+2} h_{Z}^{-2}\right)\left\|u_{\mathcal{S}}^{*}\right\|_{H^{m-1 / 2}(\mathcal{S})},
$$

for $k \geq 2$ and $m>k+d / 2+1$ for any $u_{\mathcal{S}}^{*} \in H^{m-1 / 2}(\mathcal{S})$. Effectively, the intrinsic approach lower the smoothness requirement on the exact solution $u_{\mathcal{S}}^{*}$ by a half.

Note the presence of surface differential operator $\mathcal{L}_{\mathcal{S}}$ in $(15)$, on which some extra analytical or numerical treatments are required.

\subsection{Numerical algorithms}

Some readers may be unfamiliar with surface differential operators. We begin with a simple example to demonstrate how one can collocate $\mathcal{L}_{\mathcal{S}}$ analytically.

\subsubsection{Example}

Let $\mathcal{S} \in \mathbb{R}^{3}$ be the unit sphere. We consider the differential operator $\mathcal{L}_{\mathcal{S}}=\Delta_{\mathcal{S}}-\mathbf{1} \cdot \nabla_{\mathcal{S}}$. Firstly, we compute the orthogonal projection operator

$$
\mathcal{P}=I-\mathbf{n n}^{T}=\left[\begin{array}{ccc}
-x^{2}+1 & -x y & -x z \\
-x y & -y^{2}+1 & -y z \\
-x z & -y z & -z^{2}+1
\end{array}\right]
$$

Let $\mathcal{P}_{k}$ denotes the $k$ th column of $\mathcal{P}$. Using the fact that

$$
\nabla_{\mathcal{S}} u=\mathcal{P} \nabla u=\left[\begin{array}{c}
\mathcal{P}_{1} \cdot \nabla \\
\vdots \\
\mathcal{P}_{d} \cdot \nabla
\end{array}\right] u
$$


and

$$
\Delta_{\mathcal{S}} u=(\mathcal{P} \nabla) \cdot(\mathcal{P} \nabla) u=\operatorname{trace}\left(\mathcal{P} \cdot J^{T}\left(\nabla_{\mathcal{S}} u\right)\right),
$$

we can express the surface operator entirely in Cartesian coordinates after some symbolic manipulations as

$$
\begin{aligned}
\mathcal{L}_{\mathcal{S}}= & \left((x-1)^{2}+x(y+z)-2\right) \frac{\partial}{\partial x}+\left((y-1)^{2}+y(x+z)-2\right) \frac{\partial}{\partial y} \\
& +\left((z-1)^{2}+z(x+y)-2\right) \frac{\partial}{\partial z} \\
& +\left(-x^{2}+1\right) \frac{\partial^{2}}{\partial x^{2}}+\left(-y^{2}+1\right) \frac{\partial^{2}}{\partial y^{2}}+\left(-z^{2}+1\right) \frac{\partial^{2}}{\partial z^{2}} \\
& -2 x y \frac{\partial^{2}}{\partial x \partial y}-2 x z \frac{\partial^{2}}{\partial x \partial z}-2 y z \frac{\partial^{2}}{\partial y \partial z},
\end{aligned}
$$

which no longer has any implicit dependency on $\mathcal{S}$ and can be used for collocation. We can solve (15) by solving the following $n_{X} \times n_{Z}$ overdetermined system

$$
\mathcal{L}_{\mathcal{S}} \Phi_{m}(X, Z) \boldsymbol{\lambda}=f(X)
$$

for $\boldsymbol{\lambda}$ in the least-squares sense. The numerical solution is given as in (11).

The above analytic approach works as long as we have analytic formula for the normal vector $\mathbf{n}$ of the surface, which is the case when $\mathcal{S}$ is given by some parametric formula or level set method. In cases when this is not so, i.e., when $\mathcal{S}$ is defined by a point cloud, we can opt for a pseudospectral approximation [16] and here is how.

We assume $\mathbf{n}(z)$, for all $z \in Z$, is known either a priori or by some approximations [17], [18]. Without explicitly knowing the function values of any surface function $u_{\mathcal{S}}$ at $Z$, we can still express the interpolant $I_{Z} u$ of $u$ in the trial space $\mathcal{U}_{Z, \mathcal{S}, \Psi_{m-1 / 2}}$ as

$$
I_{Z} u_{\mathcal{S}}=\left[\Psi_{m-1 / 2}(\cdot, Z)\right]\left[\Psi_{m-1 / 2}(Z, Z)\right]^{-1} u_{\mathcal{S}}(Z) .
$$

Since $Z \subset \mathcal{S}$, we have $\Psi_{m-1 / 2}(Z, Z)=\Phi_{m}(Z, Z)$ and no special treatment is required on the global kernel. The idea of pseudospectral is to approximate derivatives of $u_{\mathcal{S}}$ by that of $I_{Z} u_{\mathcal{S}}$. For surface gradient, we have

$$
\nabla_{\mathcal{S}} u_{\mathcal{S}} \approx \widetilde{\nabla}_{\mathcal{S}} u_{\mathcal{S}}:=\nabla_{\mathcal{S}}\left(I_{Z} u_{\mathcal{S}}\right)=\left[\begin{array}{c}
\mathcal{P}_{1} \cdot \nabla \\
\vdots \\
\mathcal{P}_{d} \cdot \nabla
\end{array}\right]\left(I_{Z} u_{\mathcal{S}}\right)
$$

One can, see [16] for details, write the $k$ th component of $\nabla_{\mathcal{S}}\left(I_{Z} u_{\mathcal{S}}\right)$ in the form of

$$
\left[\widetilde{\nabla}_{\mathcal{S}} u_{\mathcal{S}}\right]_{k}=\underbrace{\left(\mathcal{P}_{k}^{T}\left[\nabla \Phi_{m}(\cdot, Z)\right]\left[\Phi_{m}(Z, Z)\right]^{-1}\right)}_{=: G_{k}(\cdot, Z)} u_{\mathcal{S}}(Z) .
$$

We need all these $1 \times n_{Z}$ vector function $G_{k}$ to apply another round of pseudospectral for the surface Laplacian operator, namely

$$
\Delta_{\mathcal{S}} u_{\mathcal{S}} \approx \widetilde{\nabla}_{\mathcal{S}} \cdot \nabla_{\mathcal{S}}\left(I_{Z} u_{\mathcal{S}}\right)(Z)
$$


in which the gradient of $u_{\mathcal{S}}$ is approximated, then evaluated, at $Z$. This specific choice allows simplification for a simple formula

$$
\widetilde{\Delta}_{\mathcal{S}} u_{\mathcal{S}}=\sum_{k=1}^{d} G_{k}(\cdot, Z) G_{k}(Z, Z) u_{\mathcal{S}}(Z) .
$$

At this point, we can collocate at some set of collocation points $X \subset \mathcal{S}$ to yield an $n_{X} \times n_{Z}$ overdetermined matrix system

$$
\left[a \sum_{k=1}^{d} G_{k}(X, Z) G_{k}(Z, Z)+\mathbf{b}^{T} G(X, Z)+c \Phi_{m}(X, Z) \Phi_{m}^{-1}(Z, Z)\right] u_{\mathcal{S}}(Z)=f(X)
$$

for unknown $u(Z)$. By the relationship $\boldsymbol{\lambda}=\Phi_{m}^{-1}(Z, Z) u_{\mathcal{S}}(Z)$, which appears in all terms in the form of $G_{k}(\cdot, Z) u_{\mathcal{S}}(Z)$, we can cast the system in terms of unknown $\lambda$ easily.

\section{NUMERICAL EXAMPLES}

We provide some numerical demonstrations using the (global) Whittle-Matérn-Sobolev kernels of order $m$ as in (8). The first example will provide readers a sense of convergence of the methods in this paper. The next two aim to show how the methods can be applied in other applications, namely, solving surface pattern formation problems and convection-diffusion equations on moving surfaces. We denote our methods as follows:

Method 1: Embedding Kansa method as in (13),

Method 2: Approximated collocation method as in (19),

Method 3: Direct collocation analytically as in (17).

Sets of nodes $X$ and $Z$ are both quasi-uniform with some oversampling ratio $h_{Z} / h_{X}$ based on fill distances. Having a specific number of points quasi-uniformly distributed on a surface is not an easy task that worths to be solved exactly. In our computations, we simply enforce $h_{Z}=\gamma h_{X}$ so that the oversampling ratio $\gamma$ is roughly equal to some preassigned value.

\subsection{Convergence rates and accuracy}

We solve the surface elliptic PDE (2) with $a_{\mathcal{S}}=c_{\mathcal{S}}=1$ and $\mathbf{b}_{\mathcal{S}}=\mathbf{0}$. Let the exact solution be

$$
u_{\mathcal{S}}^{*}=(x+y+z) \exp (-x(x-1)-y(y-1)+z)
$$

defined on a constant distance product (CPD) surface

$$
\begin{aligned}
& \sqrt{(x-1)^{2}+y^{2}+z^{2}} \sqrt{(x+1)^{2}+y^{2}+z^{2}} \\
& \cdot \sqrt{x^{2}+(y-1)^{2}+z^{2}} \sqrt{x^{2}+(y+1)^{2}+z^{2}}=1.1 .
\end{aligned}
$$

We apply all methods with an oversampling ratio of $\gamma \approx 1.4$. Fig. 1 shows the error distributions on the surface obtained by Method 3 with $m=4$. In Fig. 2 , the $L^{2}(\mathcal{S})$ and $H^{2}(\mathcal{S})$ error profiles with the kernels of order $m=k+d / 2+1 / 2=4$ and $m=k+d / 2+$ $3 / 2=5$ are plotted respectively. Note that the former $m$ is below what error estimates (12) and (16) call for. All subfigures were made with the same scale for easy comparison. 

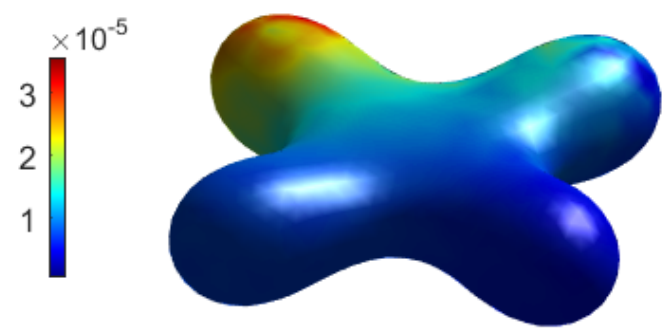

Figure 1: Error functions, obtained by Method 2 with the oversampling collocations: $h_{Z} \approx$ $1.4 h_{X} \approx 0.02$ and kernels of smoothness order $m=4$.

Clearly, kernels with smoothness order below the theoretical requirement for the error estimates, i.e., $m=4$, remain practical. In terms of accuracy, errors of Method 3 the best among the three. With our smooth solution, larger value of $m$ is also beneficial to all methods in terms of accuracy. The $H^{2}(\mathcal{S})$-error convergence rates of Method 1 are close to the predicted ones $(m-2.5)$ in theory. Though the theoretical rate $(m-3.5)$ for Method 3 is lower compared with Method 1, it achieves higher orders of convergence in this example. Convergence rate of Method 2 sits between those of both approaches, which justifies our choice of approximation methods to the surface differential operators.

\subsection{Pattern formation on surfaces}

In this section, we solve a system of coupled reaction-diffusion equations for pattern formations on surfaces. In general, a specific pattern can be obtained by solving the following equations:

$$
\left\{\begin{array}{l}
\frac{\partial u}{\partial t}=\delta_{u} \Delta_{\mathcal{S}} u+f_{u}(u, v) \\
\frac{\partial v}{\partial t}=\delta_{v} \Delta_{\mathcal{S}} v+f_{v}(u, v)
\end{array}\right.
$$

where $u$ and $v$ are the activator and inhibitor of the system. In particular, we select

$$
\left\{\begin{array}{l}
f_{u}(u, v)=\alpha u\left(1-\tau_{1} v^{2}\right)+v\left(1-\tau_{2} u\right), \\
f_{v}(u, v)=\beta v\left(1+\frac{\alpha \tau_{1}}{\beta} u v\right)+u\left(\gamma+\tau_{2} v\right),
\end{array}\right.
$$

as the reaction terms in (20). In this numerical experiment, we generate two different patterns on a Torus Knot. The non-linear problem (20) is solved by Method 1, which replaces the surface Laplacian operator with traditional Laplacian with two additional constraints. After converting the surface PDE to its embedding space, we solve the embedded PDE by a secondorder semi-implicit backward differentiation formula (SBDF2) until the solution reaches steady state. In Fig. 3, we show the steady-state solutions of (20) with two different sets of parameters $\left(\alpha, \beta, \tau_{1}, \tau_{2}\right)$; one for a spot pattern and the other for some stripe pattern. It is convincing that our method is a robust alternative for pattern formation problems. The simulation was implemented by MATLAB parallel computing toolbox. The computation time using a consumer-graded NVIDIA GeForce GTX 1080 took around 204 seconds to attain its 
(a) $m=4$
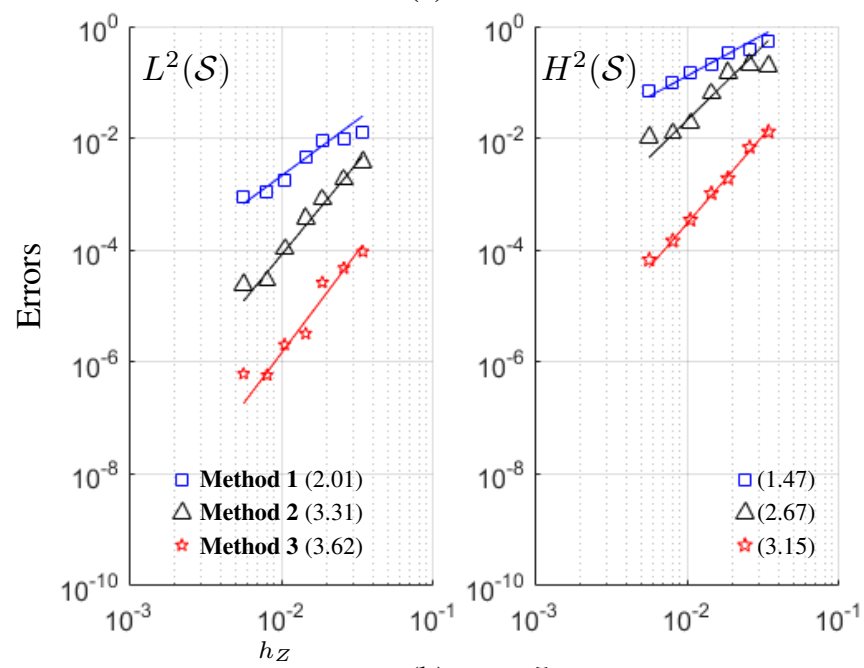

(b) $m=5$
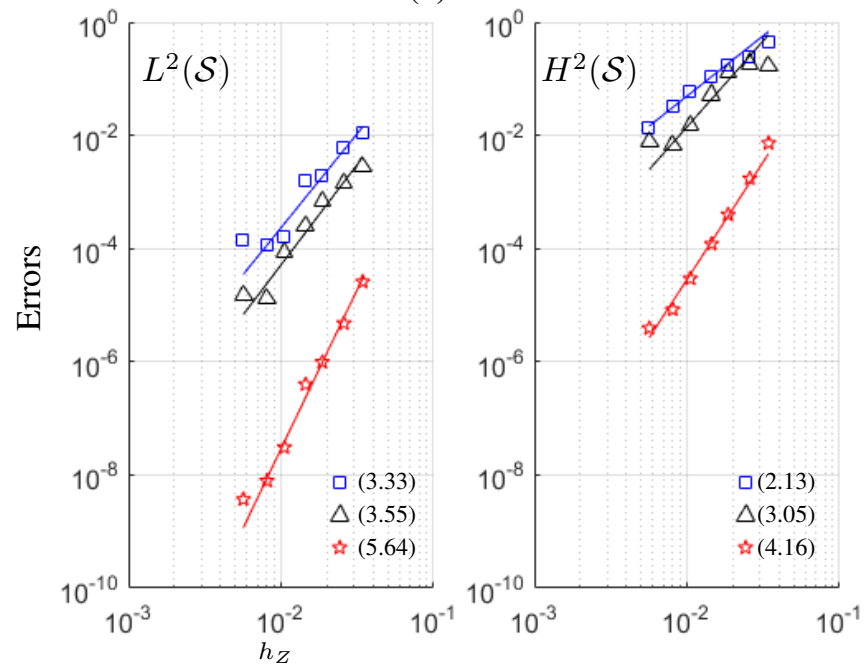

Figure 2: For the kernels of (a) $m=4$ and (b) $m=5$, the $L^{2}(\mathcal{S})$ and $H^{2}(\mathcal{S})$ error profiles with convergence rates listed in legends by using our three methods, based on the same seven pairs of $X$ and $Z\left(h_{Z} \approx 1.4 h_{X}\right)$.

steady state solution while it took 4228 seconds on CPU. Such 20X speed-up is done simply by adding few lines of codes to convert the system matrix to GPU Array.

\subsection{Merging surfaces by curvature motion}

Let the evolving surface with time $t \in[0, T]$ be denoted as $\mathcal{S}(t)$. In this test, initial surfaces are two spheres of radius 0.72 on the upper left side and 1.8 on the lower right side, shown in Fig. 4. First, we let they approach to each other at velocities $\mathbf{v}=\frac{\sqrt{2}}{2}(1,0,-1)$ and $-\mathbf{v}$ respectively. Then they collide at $t=0.01$ to generate a new closed and smooth surface, see 

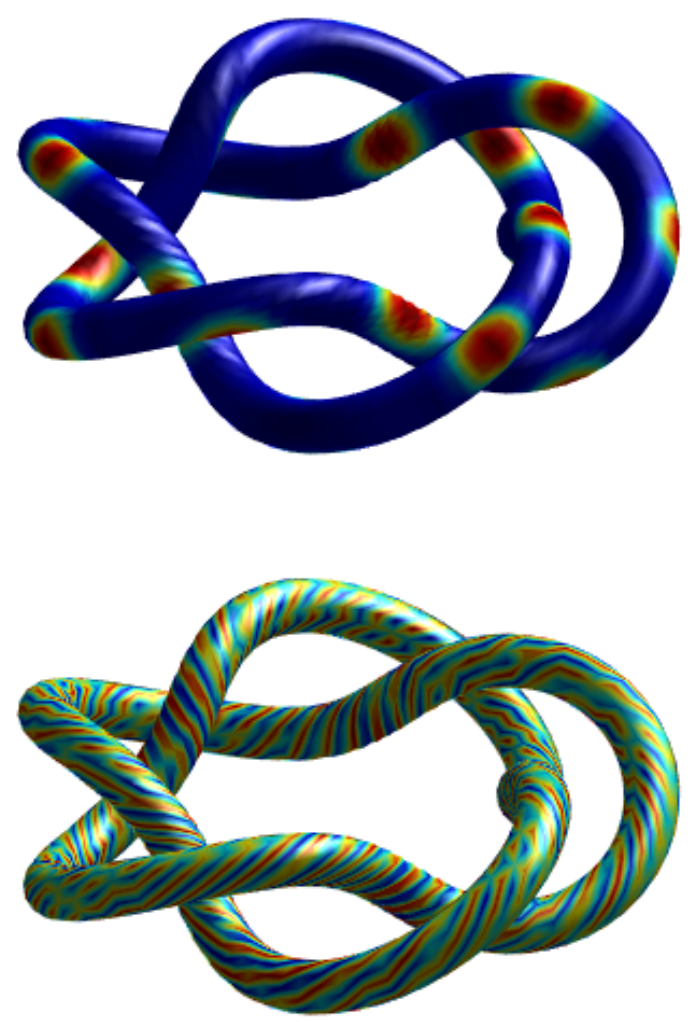

Figure 3: Steady state solutions of the reaction diffusion equation obtained by Method 1.

Fig. 5(a). The merged surface further evolved under geometric motion $\mathbf{v}=-\kappa \mathbf{n}$ by the mean curvature $\kappa=\nabla_{\mathcal{S}} \cdot \mathbf{n}$, see [19]. Along the evolution, we compute point clouds by the method in [20].

Now, on $\mathcal{S}(t)$, we want to solve convection-diffusion equations

$$
\partial_{t} u+\mathbf{v} \cdot \nabla u+\left(\nabla_{\mathcal{S}} \cdot \mathbf{v}\right) u-\Delta_{\mathcal{S}} u=0 \quad \text { on } \mathcal{S}(t) \times[0, T]
$$

subject to some Dirichlet initial conditions. In (22), vector $\mathbf{v}$ denotes the velocity of surface motion.

We first discretize (22) by the Crank-Nicolson (CN) method in time. Then, we apply Method 2 in space to solve the discretized problem and complete the time stepping. Note that Method 3 is not appropriate for this problem due to the lack of analytical information. Implementing Method 1 also involves certain difficulties, e.g., the high curvature occurring right after the merge poses a constraint and we must use some very narrow-band domains.

At each time step before merging $(t<0.01)$, we respectively solve the problem (22) on both spheres, via our oversampled Method 2 to the time semi-discretized problem. We employ kernels of low order of smoothness $m=2.5$ and the time step size is set to be $\Delta t=0.001$. At the moment of merging at $t=0.01$, the numerical solutions at merged points are obtained by 


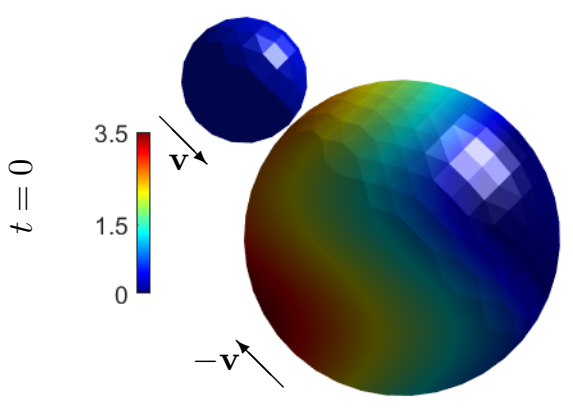

Figure 4: The initial conditions (color) with $n_{X}=567, n_{Z}=365$ totally on two merging different spheres at constant velocities $\mathbf{v}=\frac{\sqrt{2}}{2}(1,0,-1)$ and $-\mathbf{v}$ (arrow).

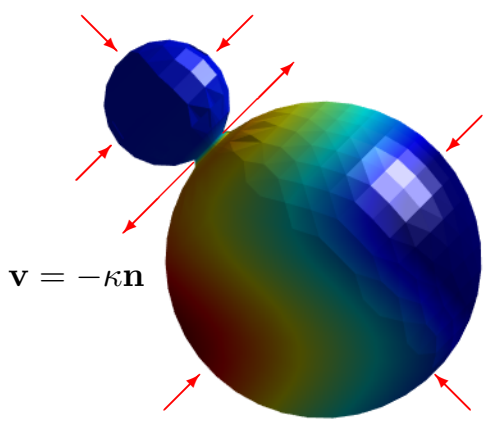

(a) $t=0.01$

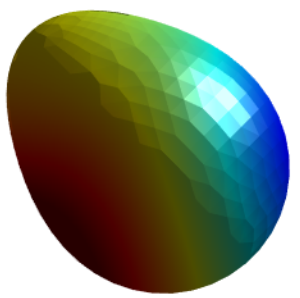

(c) $t=0.6$

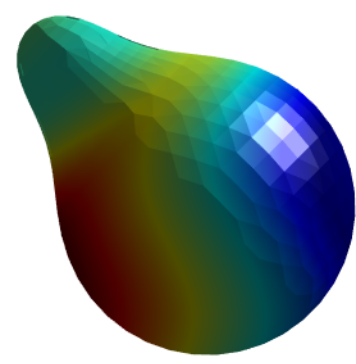

(b) $t=0.2$

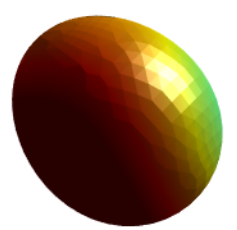

(d) $t=1$

Figure 5: The corresponding snapshots of numerical solutions (color) with evolving velocity (arrow) at several time points, obtained by Method 3 with $\mathrm{CN}$, using $n_{X}=666$, $n_{Z}=427, m=2.5$ and $\triangle t=10^{-3}$ with the same scalars of color and coordinates as in Fig. 4, on a mean-curvature evolving surface.

interpolation in Fig. 5(a). After then, we use approximated normal vectors to simulate meancurvature motion. Numerical results of (22) obtained by Method 3 at three times $t=0.2,0.6$ and 0.1 are color mapped in Fig. 5(b)-(d) respectively. 


\section{CONCLUSION}

This paper reviews three alternatives for solving surface PDEs. We focus on surfaces with codimension one for simplicity; all methods in this paper can be easily generalized to higher codimensions. In our presented example, we see some higher than predicted convergence rates, which is not uncommon to see in Kansa-type methods for surface PDEs. Generally speaking, convergence rate will gradually slow down to the predicted one as the surface curvature increases. Although all presented methods are global (because we have rigorous convergence theorems), we want to point out to our readers that there are many local meshfree methods that solve surface PDEs. Readers are recommended to include those in consideration before pinpointing their method of choice.

\section{REFERENCES}

[1] Strichartz, R.S., Analysis of the Laplacian on the complete Riemannian manifold. $J$. Funct. Anal., 52, pp. 48-79, 1983.

[2] Wloka, J., Partial Differential Equations. Cambridge University Press, 1987.

[3] Kansa, E.J., Application of Hardy's multiquadric interpolation to hydrodynamics. Proceedings of the 1986 Simulation Conference, vol. 4, pp. 111-117, 1986.

[4] Kansa, E.J., Multiquadrics - a scattered data approximation scheme with applications to computational fluid-dynamics. I. Surface approximations and partial derivative estimates. Comput. Math. Appl., 19(8-9), pp. 127-145, 1990.

[5] Ruuth, S.J. \& Merriman, B., A simple embedding method for solving partial differential equations on surfaces. J. Comput. Phys., 227(3), pp. 1943-1961, 2008.

[6] Piret, C., The orthogonal gradients method: A radial basis functions method for solving partial differential equations on arbitrary surfaces. J. Comput. Phys., 231(14), pp. 46624675, 2012.

[7] Chen, Y. \& Macdonald, C.B., The closest point method and multigrid solvers for elliptic equations on surfaces. SIAM J. Sci. Comput., 37(1), pp. A134-A155, 2015.

[8] Cheung, K.C. \& Ling, L., A kernel-based embedding method and convergence analysis for surfaces PDEs. SIAM J. Sci. Comput., 40(1), pp. A266-A287, 2018.

[9] Wendland, H., Error estimates for interpolation by compactly supported radial basis functions of minimal degree. J. Approx. Theory, 93(2), pp. 258-272, 1998.

[10] Buhmann, M.D., Radial basis functions: Theory and implementations, vol. 12 of Cambridge Monographs on Applied and Computational Mathematics. Cambridge University Press: Cambridge, 2003.

[11] Wendland, H., Scattered data approximation, vol. 17 of Cambridge Monographs on Applied and Computational Mathematics. Cambridge University Press: Cambridge, 2005.

[12] Cheung, K.C., Ling, L. \& Schaback, R., $H^{2}$-convergence of least-squares kernel collocation methods. SIAM J. Numer. Anal., 56(1), pp. 614-633, 2018.

[13] Fuselier, E.J. \& Wright, G.B., Scattered data interpolation on embedded submanifolds with restricted positive definite kernels: Sobolev error estimates. SIAM J. Numer. Anal., 50(3), pp. 1753-1776, 2012.

[14] Narcowich, F.J., Sun, X. \& Ward, J.D., Approximation power of RBFs and their associated SBFs: A connection. Adv. Comput. Math., 27(1), pp. 107-124, 2007.

[15] Chen, M. \& Ling, L., Intrinsic meshless collocation methods for PDEs on manifolds. SIAM J. Num. Anal., to be published.

[16] Fuselier, E.J. \& Wright, G.B., A high-order kernel method for diffusion and reactiondiffusion equations on surfaces. J. Sci. Comput., 56, pp. 535-565, 2013. 
170 Boundary Elements and other Mesh Reduction Methods XLII

[17] Fornberg, B., Driscoll, T.A., Wright, G. \& Charles, R., Observations on the behavior of radial basis function approximations near boundaries. Comput. Math. Appl., 43(3-5), pp. 473-490, 2002.

[18] Marchandise, E., Piret, C. \& Remacle, J.F., CAD and mesh repair with radial basis functions. J. Comput. Phys., 231(5), pp. 2376-2387, 2012.

[19] Deckelnick, K., Dziuk, G. \& Elliott, C.M., Computation of geometric partial differential equations and mean curvature flow. Acta Numer., 14, pp. 139-232, 2005.

[20] Bronsard, L. \& Wetton, B.T.R., A numerical method for tracking curve networks moving with curvature motion. J. Comput. Phys., 120(1), pp. 66-87, 1995. 